\title{
Transmission Control for Compressive Sensing Video over Wireless Channel
}

\author{
Siyuan Xiang, Student Member, IEEE, and Lin Cai, Senior Member, IEEE
}

\begin{abstract}
In this paper, we consider a wireless sensor node monitoring the environment and it is equipped with a compressive-sensing based, single-pixel image camera and other sensors such as temperature and humidity sensors. The wireless node needs to send the data out in a timely and energy efficient way. This transmission control problem is challenging in that we need to jointly consider perceived video quality, quality variation, power consumption and transmission delay requirements, and the wireless channel uncertainty. We address the above issues by first building a rate-distortion model for compressive sensing video. Then we formulate the deterministic and stochastic optimization problems and design the transmission control algorithm which jointly performs rate control, scheduling and power control. Extensive simulations have been conducted to demonstrate the effectiveness of the proposed transmission control algorithm.
\end{abstract}

Index Terms-Compressive sensing video, stochastic optimization, rate-distortion, video transmission.

\section{INTRODUCTION}

C OMPRESSIVE sensing has been an active research area in signal processing and communication societies recently. The joint acquisition and compression framework and the characteristics of the acquired measurements attract many applications in image and video acquisition and also raise many new challenges.

Specifically, unlike traditional transform domain compression method which acquires the complete image signal then compress the signal by removing the redundancy, Compressive Sensing (CS) unifies these two operations by making random linear projection of the source signals. The acquired measurements have special property referred as "democracy", i.e., the measurements are equally important and the more measurements generated, the better quality of the recovered image signal. This characteristic is promising to make the image and video bitstream more error resilient and scalable. How to fully utilize this characteristic of CS measurements is still an open issue.

We have seen the first application of CS in image acquisition single-pixel image camera [1]. Thanks to its simplicity and less power requirement, it has a good potential to be widely deployed in wireless sensor nodes. In this paper, we consider a wireless sensor node monitoring the environment and it is equipped with a single-pixel image camera and some other sensors such as temperature and humidity sensors. The sensor

Manuscript received August 6, 2012; revised November 8, 2012; accepted December 21, 2012. The associate editor coordinating the review of this paper and approving it for publication was S. Mao.

The authors are with the Department of Electrical and Computer Engneering, University of Victoria, Victoria, British Columbia, Canada (e-mail: \{siyxiang, cai\}@ece.uvic.ca).

Digital Object Identifier 10.1109/TWC.2013.012313.121150 node needs to send the data out in a timely and energy efficient way. This is not a trivial problem which involves the following issues and requirements. 1) The first issue is rate control. The single-pixel image camera can capture the image and obtain a fixed number of measurements. We need to determine the number of measurements transmitted for each image while considering the transmission power consumption, the wireless channel condition and the recovered image quality. To achieve a better perceived video quality, we need to avoid large image quality fluctuations. 2) The second issue is scheduling. The CS acquired measurements are scalable and the traffic is elastic, while the packets from the other sensors may be non-elastic. Due to the heterogeneity, it is necessary to differentiate these two types of traffic. Therefore, we need to optimize the scheduling of packet transmissions from different traffic flows on each time slot. 3) The third issue is power control. In general, we can minimize the transmission power by sending less measurements. However, we need to take both the wireless channel conditions and the recovered image quality into consideration. 4) The last issue is the delay constraint. In order to deliver the packets within a reasonable delay, we need to keep the transmission queues stable.

Our main contributions in this paper are three-fold. First, to perform rate control, we propose a rate-distortion model to capture the relationship between the number of measurements and the recovered signal distortion based on the CS theory. We have shown the accuracy and effectiveness of the proposed model. Second, we formulate a deterministic optimization algorithm as a benchmark, and a more practical stochastic optimization problem for optimizing the rate control and power control. In addition, we propose a supplementary stochastic optimization algorithm which puts different priority in conserving power and video quality requirements. For the deterministic optimization problem, Lagrangian optimization and dynamic programming are used to obtain the optimal solution. For the stochastic optimization problem, the Lyapunov optimization technique is used to design a joint rate control, power control and scheduling algorithm. Third, we conduct extensive simulation to validate the proposed rate-distortion model and the control algorithms and show their effectiveness.

The rest of the paper is organized as follows. Section II gives a brief introduction of compressive sensing and the related work. Section III describes the system model and the formulation of the optimization problems. Section IV details the derivation of the joint rate control, power control and scheduling algorithm. The performance of the proposed ratedistortion model and control algorithm is studied in section $\mathrm{V}$, followed by concluding remarks in section VI. 


\section{BACKGROUND AND RELATED WORK}

\section{A. Background of Compressive Sensing}

Compressive sensing or sampling [2] [3] was proposed as a new acquisition framework which can sample and compress sparse or compressible signals in a single operation.

Suppose that a signal $x \in \mathbb{R}^{n}$ can be transformed to coefficients vector $\theta$ with some basis $\Psi$, i.e. $x=\Psi \theta$. $\Psi$ can be any representing basis such as DCT or wavelet. If $S$ elements of vector $\theta$ is nonzero, then $x$ is called $S$-sparse signal. If we sort the magnitude of $\theta$ in a descending order and it follows the following power-law

$$
|\theta|_{n} \leq R n^{-1 / p},
$$

where $R$ and $p$ are positive constants, the signal $x$ is compressible. $p$ determines how fast the amplitude of the coefficients decays. Image signals are usually compressible. When we use the largest $S$ elements of $\theta$, denoted as $\theta_{S}$, with all other elements set to zero to approximate $\theta$, the approximation error follows

$$
\left\|\theta-\theta_{S}\right\|_{\ell_{2}} \leq C R S^{-r}
$$

where $C$ is a constant and $r=1 / p-1 / 2$. If we increase $S$, according to (2), the approximation error decays quickly.

The $m$ measurements of compressive sensing, $y$, are obtained by multiplying signal $x$ with a measurement matrix $\Phi \in \mathbb{R}^{m \times n}$, i.e., $y=\Phi x$. Using the reverse operation to recover $x$ is infeasible, since, given $m<n, y=\Phi \tilde{x}$ is an underdetermined system with infinite solutions. However, [2] and [3] have shown that $\ell_{1}$ minimization can recover the original signal with high probability, which can be formulated as

$$
\begin{array}{ll}
\min & \|\tilde{\theta}\|_{\ell_{1}} \\
\text { subject to } & \|y-A \tilde{\theta}\|_{\ell_{2}} \leq \epsilon,
\end{array}
$$

where $A=\Phi \Psi$ and $\epsilon$ is the noise energy in the measurements. The recovered signal $x=\Psi^{*} \hat{\theta}$, where $\Psi^{*}$ is the adjoint of $\Psi$, and $\hat{\theta}$ is the solution to (3). For compressible signal, [3] provided the following theorem to bound the recovery performance.

Theorem 1: If $x$ obeys (1), with high probability

$$
\|x-\hat{x}\|_{\ell_{2}} \leq C R(m / \log n)^{-r},
$$

where $m$ is the number of measurements and $r=1 / p-1 / 2$.

We can see the relationship between (4) and (2). When $S=O(m / \log n)$, the right hand side of both equations are equivalent. It means $\|x-\hat{x}\|_{\ell_{2}}=\|\hat{\theta}-\theta\|_{\ell_{2}}$. Therefore, this theorem states that if there are $O(S \log n)$ measurements, the recovery is as good as knowing $x$ and selecting the largest $S$ coefficients from $\theta$.

\section{B. Related Work}

How to deliver traditional video over wireless networks have been heavily investigated [4]-[9]. There are a few works investigating transmitting compressive sensing measurements over wireless channel. [10] used compressive sensing as a joint source and channel coding to protect sparse signals over erasure channel. The proposed method compensates the lost measurements during transmission by sending more

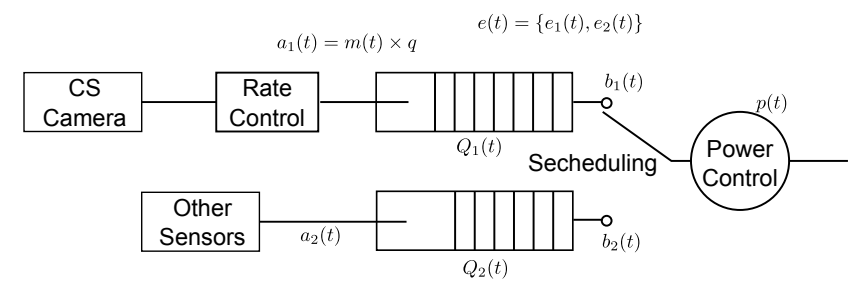

Fig. 1. System architecture.

measurements. This property is also referred as democracy of compressive sensed measurements [11].

For compressive sensing image or video rate control algorithm, the channel error/erasure rate is not the only factor to consider. [12] proposed a system for joint video compression, rate control and error correction over wireless sensor network using compressive sensing. The proposed rate control algorithm considers network congestion and received video quality. The rate control algorithm uses a rate-distortion model from a conventional video compression method [13], which may not fully capture the rate-distortion characteristic of compressive sensing measurements. In addition, it did not consider transmission power control to maximize the lifetime of wireless sensor nodes.

[14] proposed a joint compression and transmission power control algorithm for packets processing in wireless nodes. Stochastic network optimization is used to obtain the control decisions to minimize the power consumption while making the transmission queues stable. However, how to consider rate control and the perceived quality for compressive sensing video transmission is still an open issue, which motivates this work.

\section{System Model And Problem Formulation}

\section{A. System Model}

Fig. 1 shows the internal structure of the sensor node. The single-pixel image camera acquires $F$ images per second and generate $A_{\max }$ compressive sensing measurements for each image. Naturally, we divide the time into slots with a duration of $1 / F$ seconds, so the camera generates $A_{\max }$ measurements per time slot. The rate control module decides how many measurements $m(t)$ should be put into $Q_{1}$ to transmit. Each measurement is represented by $q$ bits. Therefore, the arrival rate of $Q_{1}$ is $a_{1}(t)=m(t) \times q$ and $a_{1}(t)$ is in the range of $\left(0, A_{\max } \times q\right)$ at time slot $t$. The queue backlog of $Q_{1}$ at time slot $t$ is $Q_{1}(t)$. Denote $d(t)$ as the distortion incurred when admitting $m(t)$ measurements into the queue for each image. We assume that the number of bits per measurement is fixed as $q$, so the number of measurements is the only way to adjust the video rate. Other sensed (non-video) data will be put into another queue $Q_{2}$. The queue backlog at time slot $t$ is $Q_{2}(t)$ and its arrival rate is $a_{2}(t)$ bits/slot.

We have two queues and need to select at most one queue per time slot for transmission. We denote the decision as $e(t)=\left\{e_{1}(t), e_{2}(t)\right\}$, where $e_{k}(t)=1$ means selecting queue $k$ to transmit and otherwise $e_{k}(t)=0$. When both $e_{1}(t)$ and $e_{2}(t)$ are both 0 , nothing is transmitted, and we have $e_{1}(t)+e_{2}(t) \leq 1$. Define $b_{k}(t)$ as the service rate 
for queue $k$. It is a function of $e(t)$ and the power level $p(t)$, where $0 \leq p(t) \leq p_{\max }$ and $p_{\max }$ is the maximum transmission power. $p(t)=0$ indicates that $e_{1}(t)+e_{2}(t)=0$ and vice versa. The service rate according to channel capacity is approximately

$$
b_{k}(t)=\frac{\eta B}{F} e_{k}(t) \log \left(1+p(t) \frac{d^{-\alpha} G}{N_{0} B}|h(t)|^{2}\right)(\text { bits/slot })
$$

where $B$ is the channel bandwidth $(\mathrm{Hz}), \eta$ is a communication system coefficient representing the transceiver efficiency, $d$ is the distance between the transmitter and receiver, $\alpha$ is the path-loss exponent and $h(t)$ is the fading coefficient at time slot $t$. We assume that $h(t)$ is known at the beginning of each slot. We need to determine the power level $p(t)$ for each time slot. The queue dynamics is

$$
Q_{k}(t+1)=\max \left[Q_{k}(t)-b_{k}(t), 0\right]+a_{k}(t) .
$$

\section{B. Rate-Distortion Model}

Before we formulate the optimization problem, we need to build a rate-distortion model for compressive sensed video. According to (4), $\ell_{1}$-minimization is as good as recovering the largest $S$ non-zero coefficients. We define the rate distortion model as follows

$$
D(m)=\alpha m^{\beta},
$$

where $\mathrm{D}(\mathrm{m})$ denotes the mean square distortion function, $m$ is the number of measurements, $\alpha$ and $\beta$ are the parameters of this model. Note that for notation simplicity, $m=a(t)$ and $D(m)=d(t)$. To better approximate the rate-distortion relation, we use following model with one more parameter,

$$
D(m)=\alpha m^{\beta}+\gamma .
$$

In practical, we can determine the parameters of the model online by decoding a few sets of measurements and calculating the distortion, and then use these measurement-distortion data to fit the model and obtain the parameters that can be used for a number of consecutive frames.

\section{Deterministic Optimization Problem}

We first formulate the optimal scheduling, power and rate control as a deterministic optimization problem assuming the channel condition $h(t)$ and arrival rate $a_{2}(t)$ are known. The knowledge about the channel and arrival rate are difficult to obtain in reality. But it can serve as a benchmark and its performance can be used as an upper bound for comparison purpose. The deterministic optimization problem can be decomposed to a power control problem (P1) and a rate control problem (P2). If we can transmit the maximum amount of data within time period $T$, then we have a chance to maximize the perceived video quality, since the distortion decreases as the number of transmitted measurements increases. Therefore, we first formulate and solve problem (P1), which finds an optimal power allocation scheme to maximize the amount of data can be transmitted during $T$. Then, we formulate and solve rate allocation problem $(\mathrm{P} 2)$ to minimize the weighted sum of distortion and distortion variation subject to the maximum amount of data constraint. The details of these two deterministic optimization problems are as follows.

The objective of the power control problem is to allocate total power budget $\bar{p} \times T$ among slots 0 to $T-1$ to maximize the total transmission rate, where $\bar{p}$ is the maximum average power consumption and $T$ is the number of total transmission slots. The power control problem is formally defined as follows.

$$
\begin{aligned}
\text { (P1) maximize } & \sum_{t=0}^{T-1} b(t), \\
\text { subject to } & \sum_{t=0}^{T-1} p(t) \leq T \times \bar{p}, \\
& 0 \leq p(t) \leq p_{\max },
\end{aligned}
$$

where $b(t)$ is the aggregated transmission rate of the two queues. By solving the above optimization problem, we can obtain the power allocation $p(t)$ for every slot and the maximum amount of data for $T$ slots $b^{*}=\sum_{t=0}^{T-1} b^{*}(t)$.

The objective of the rate control problem is to decide the number of measurements per slot, $m(t)$, to minimize the weighted sum of distortion and distortion variation. The problem is formulated as follows.

$$
\begin{aligned}
\text { (P2) minimize } & \sum_{t=0}^{T-1} d(t)+\mu(d(t)-d(t-1))^{2}, \\
\text { subject to } & \sum_{t=0}^{T-1}\left(a_{2}(t)+m(t) \times q\right) \leq b^{*}, \\
& 0 \leq m(t) \leq A_{\max },
\end{aligned}
$$

where $\mu$ is the weighting parameter of distortion and distortion variation. The inequality (13) assures that all data arrived during $T$ time slots can be transmitted. Thus the queues are stable.

\section{Stochastic Optimization Problem}

We next formulate two more practical stochastic optimization problems. First, we formulate the stochastic problem $(P 3)$ (power constraint) corresponding to the above deterministic problem. We aim to minimize the time average expected weighted sum of distortion and distortion variation, while keeping all the queues mean-rate stable, and maintaining the time average expected power consumption to be lower than a threshold. The optimization problem is formally defined as follows.

$$
\begin{gathered}
\text { (P3) minimize } \lim _{t \rightarrow \infty} \sup \frac{1}{t} \sum_{\tau=0}^{t-1} \mathbb{E}\left\{d(\tau)+\mu(d(\tau)-d(\tau-1))^{2}\right\} \\
\text { subject to } \lim _{t \rightarrow \infty} \frac{\mathbb{E}\left\{Q_{k}(t)\right\}}{t}=0 \text { for all } k, k=1,2 \\
\\
\lim _{t \rightarrow \infty} \sup \frac{1}{t} \sum_{\tau=0}^{t-1} \mathbb{E}\{p(\tau)\}-\bar{p} \leq 0 \\
e_{k}(t)=\{0,1\}, e_{1}(t)+e_{2}(t) \leq 1 \\
0 \leq m(t) \leq A_{\max }, 0 \leq p(t) \leq p_{\max }
\end{gathered}
$$

where $\bar{p}$ is the maximum average power consumption and $\mu$ is the parameter to set the weights of distortion and distortion 
variation. Note that $h(t), a_{2}(t), p(t)$ and $d(t)$ are time dependent random variables. (16) requires that all queues are meanrate stable. The time average expectation (15) and (17) can be viewed as the objective function (12) and inequality (10) divided by $T$ and take limsup of $T$ to infinity, respectively. Since for deterministic optimization algorithm, $b(t)$ and $d(t)$ are functions of deterministic values of $h(t)$ and $a_{2}(t)$ and the expectation of a deterministic value is itself. In addition, these two optimization problems both require that the queues should be stable. Thus, these two problems are equivalent.

Alternatively, if the requirement of the received video quality is more stringent, we can define the second optimization problem $(P 4)$ (distortion constraint) as follows.

$$
\begin{gathered}
\text { (P4) minimize } \lim _{t \rightarrow \infty} \sup \frac{1}{t} \sum_{\tau=0}^{t-1} \mathbb{E}\{p(\tau)\} \\
\text { subject to } \lim _{t \rightarrow \infty} \frac{\mathbb{E}\left\{Q_{k}(t)\right\}}{t}=0 \text { for all } k, k=1,2 \\
\lim _{t \rightarrow \infty} \sup \frac{1}{t} \sum_{\tau=0}^{t-1} \mathbb{E}\{d(\tau)\}-\bar{d} \leq 0 \\
\lim _{t \rightarrow \infty} \sup \frac{1}{t} \sum_{\tau=0}^{t-1} \mathbb{E}\left\{(d(\tau)-d(\tau-1))^{2}\right\} \\
\quad-\bar{d}_{v} \leq 0 \\
e_{k}(t)=\{0,1\}, e_{1}(t)+e_{2}(t) \leq 1 \\
0 \leq m(t) \leq A_{\max }, 0 \leq p(t) \leq p_{\max }
\end{gathered}
$$

where $\bar{d}$ is the maximum expected video distortion and $\overline{d_{v}}$ is the maximum expected distortion variation. In this problem, we aim to minimize the power consumption while keeping all the queues stable and satisfying the minimum video quality requirement. In the above two problems, we need to perform rate control which determines $m(t)$, scheduling which determines $e_{k}(t)$, and power control which determines $p(t)$ for each time slot.

\section{TRANSMisSion CONTROL AlgORITHMS}

\section{A. Deterministic Optimization Algorithm}

For the deterministic optimization problem, we first solve the power control problem followed by the rate control. We can use the Lagrangian optimization technique to rewrite the problem as

$$
\operatorname{minimize} \sum_{t=0}^{T-1}-b(t)+\lambda \sum_{t=0}^{T-1} p(t) .
$$

By [15], we can minimize $b(t)$ for each time slot independently with common $\lambda$. The algorithm is detailed in procedure PowerControl in Alogrithm 1. Basically, the algorithm increases $\lambda$ from 0 until the total power reduces to the total power budget. After each iteration, $\lambda$ is increased by a constant value $a>0$. In line $6, p(t)$ is set to the value which makes the gradient of $-b(t)+\lambda p(t)$ to 0 . When the algorithm terminates, we can obtain optimal $p^{*}(t)$ and $b^{*}(t)$ which in turn can be used to obtain the maximum transmission rate and solve the rate control problem. Dynamic programming has been used in rate allocation in conventional video coding [16].

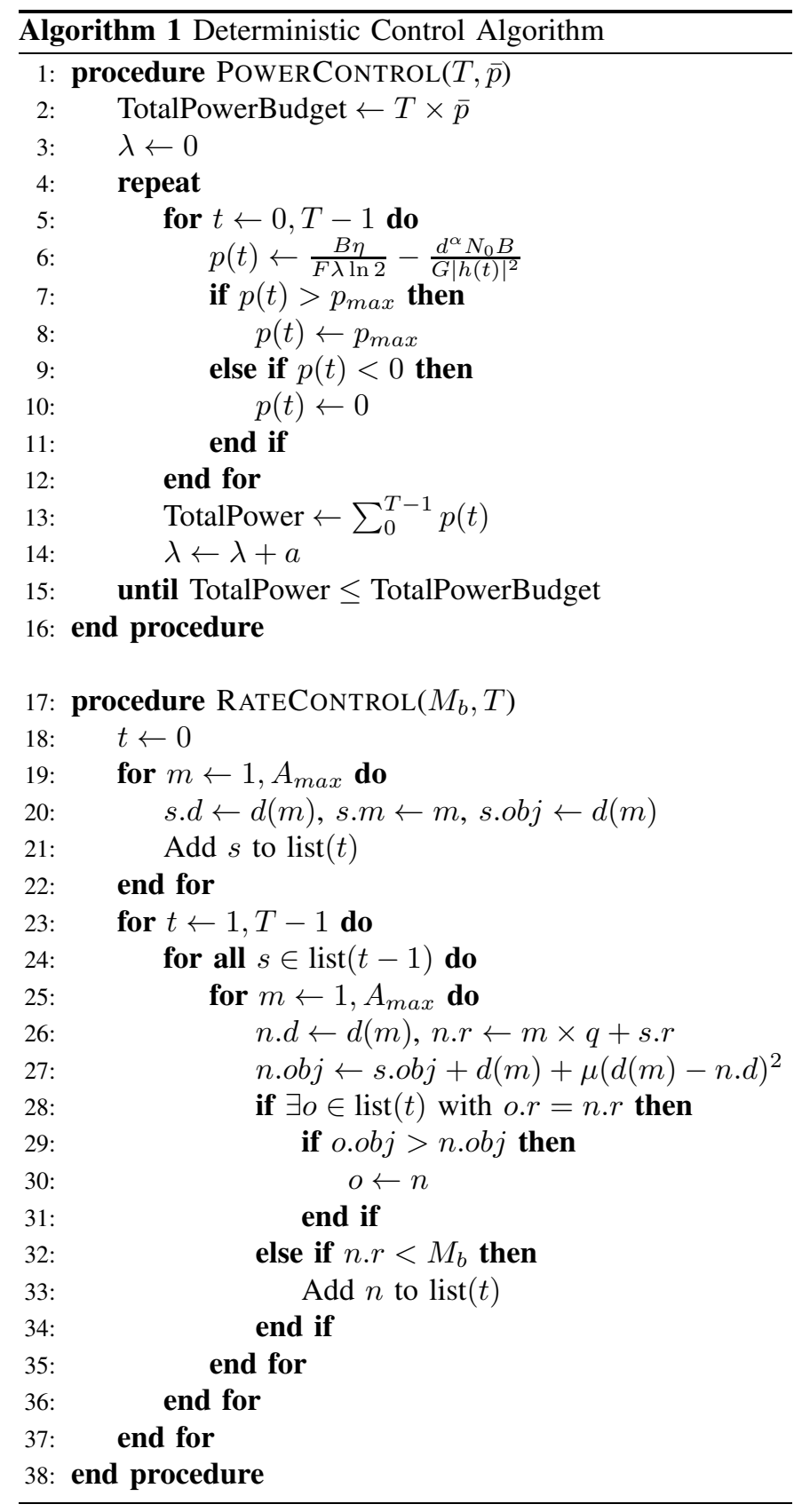

We use a dynamic programming algorithm to obtain the optimal solution. The total amount of transmission rate is known, so the total amount of data left to the measurements is $M_{b}=b^{*}-\sum_{t=0}^{T-1} a_{2}(t)$. The algorithm is detailed in procedure RateControl in Algorithm 1. The algorithm maintains a list of possible rate allocation nodes for each time slot. Each node of time slot $t$ represents a rate allocation decision from time slot 0 to $t$. A node has a few attributes to describe the rate allocation decision: $o b j$ is the accumulated weighted sum of distortion and distortion variation; $r$ is the accumulated sum of rates; $d$ is the distortion of the current time slot. Lines 19-21 initialize the nodes of time slot 0 . Lines 23-37 iterate all the time slots from 1 to $T-1$ and maintains a node list for each time slot. Lines 28-34 keep the nodes with the smaller objective value when two nodes has the same accumulated rate, and remove nodes whose rates exceeding the target rate budget $M_{b}$. When 
the algorithm terminates, we can find an optimal path of length $T$ which minimizes the objective function.

We designed a simple transmission strategy to ensure that the optimal transmission rate can be fully utilized. Specifically, we delay the transmission of all the data by $T$ slots, thus before transmission, all the data generated during $T$ slots are buffered in the queues. Then it takes another $T$ slots to transmit all the buffered data, since the total amount of transmission rate during the second $T$-slots duration and the total arrival in the first $T$-slots duration satisfy inequality (13). In this case, the scheduling problem becomes simple, and we can arbitrarily select any queue to transmit so long as the queue is nonempty and the transmission rate can be fully utilized. The transmission delay of this simple transmission strategy is $2 T$. When $T$ approaches infinity, the delay goes to infinity as well. The queues are stable, since the average arrival rate is less than the average transmission rate.

\section{B. Lyapunov Optimization Algorithm}

The deterministic control can provide an upper-bound for the transmission control performance, but it is not realistic as it requires the complete knowledge of future channel condition $h(t)$ and the arrival rate $a_{2}(t)$, and the computation complexity of the rate control algorithm is high. Therefore, we mainly focus on the stochastic optimization problems (P3) and (P4) and use Lyapunov optimization technique to solve them. In the stochastic optimization problem formulation, $h(t)$ and $a_{2}(t)$ for different time slots are considered as i.i.d. random variables and so are their functions $p(t)$ and $d(t)$.

Lyapunov optimization technique is simple to implement (no curse of dimensionality) and does not require the complete knowledge of $h(t)$ and $a_{2}(t)$ or their statistics but only the $h(t)$ of the current time slot which can be estimated using the feedback from the receiver. In addition, the performancedelay tradeoff has been proved to be $O(1 / V)$ and $O(V)$ while ensuring the queue stability [17], which shows explicit convergence of the algorithm. That is, the solution can be within $O(1 / V)$ from the optimal solution for queue stability, while the average queue backlog and delay is $O(V)$.

To solve problem (P3), we define $y(t)=p(t)-\bar{p}$ and a virtual queue $Z(t)$ as follows

$$
Z(t+1)=\max [Z(t)+y(t), 0] .
$$

If we can make the virtual queue stable, then the inequality constraint (17) can be satisfied [17]. For completeness, we present the proof here. By sample path property, we have

$$
\frac{Z(t)}{t}-\frac{Z(0)}{t} \geq \frac{1}{t} \sum_{\tau=0}^{t-1} y(\tau)
$$

Then, taking expectation and limit sup of $t$ to infinity, we can obtain

$$
\lim _{t \rightarrow \infty} \sup \frac{\mathbb{E}\{Z(t)\}}{t} \geq \lim _{t \rightarrow \infty} \sup \frac{1}{t} \sum_{\tau=0}^{t-1} \mathbb{E}\{y(\tau)\} .
$$

If the virtual queue $Z(t)$ is mean rate stable, then the lefthand-side of (28) is 0 and the right-hand-side of (28) is less than or equal to 0 . I.e.,

$$
\lim _{t \rightarrow \infty} \sup \frac{1}{t} \sum_{\tau=0}^{t-1} \mathbb{E}\{p(\tau)\}-\bar{p} \leq 0 .
$$

Let $\Theta(t)=\left[Q_{1}(t), Q_{2}(t), Z(t)\right]$ be the concatenated vectors of actual queues and virtual queue. The Lyapunov function and one-slot conditional Lyapunov drift are defined as

$$
\begin{aligned}
& L(\Theta(t))=\frac{1}{2} \sum_{k=1}^{2} Q_{k}(t)^{2}+\frac{1}{2} Z(t)^{2}, \\
& \Delta(\Theta(t))=\mathbb{E}\{L(\Theta(t+1))-L(\Theta(t)) \mid \Theta(t)\},
\end{aligned}
$$

respectively. According to [17, Lemma 4.6], the drift-pluspenalty is bounded as follows,

$$
\begin{aligned}
\Delta(\Theta(t)) & +V \mathbb{E}\left\{d(t)+\mu\left((d(t)-d(t-1))^{2}\right) \mid \Theta(t)\right\} \\
& \leq B+V \mathbb{E}\{d(t) \mid \Theta(t)\}+Z(t) \mathbb{E}\{y(t) \mid \Theta(t)\} \\
& +\sum_{k=1}^{2} Q_{k}(t) \mathbb{E}\left\{a_{k}(t)-b_{k}(t) \mid \Theta(t)\right\},
\end{aligned}
$$

where $V>0$ is a weighting parameter and $B$ is defined as

$$
\begin{aligned}
B & \geq \frac{1}{2} \sum_{k=1}^{2} \mathbb{E}\left\{a_{k}(t)^{2}+b_{k}(t)^{2} \mid \Theta(t)\right\}+\mathbb{E}\{y(t) \mid \Theta(t)\} \\
& -\sum_{k=1}^{2} \mathbb{E}\left\{\tilde{b}_{k}(t) a_{k}(t) \mid \Theta(t)\right\}
\end{aligned}
$$

where $\tilde{b}_{k}(t)=\min \left[b_{k}(t), Q_{k}(t)\right]$. Instead of minimizing the left-hand-side of (31), we minimize the right-hand-side bound opportunistically. The optimization problem (15) is casted to a deterministic optimization problem.

$$
\begin{aligned}
\operatorname{minimize} & V\left(d(t)+\mu(d(t)-d(t-1))^{2}\right) \\
+ & \sum_{k=1}^{2} Q_{k}(t)\left(a_{k}(t)-b_{k}(t)\right)+Z(t) y(t)
\end{aligned}
$$

subject to $e_{k}(t)=\{0,1\}, e_{1}(t)+e_{2}(t) \leq 1$,

$$
0 \leq m(t) \leq A_{\max }, \quad 0 \leq p(t) \leq p_{\max } \text {. }
$$

We can decompose the above optimization into two separate optimization problems. The first one is a rate control problem.

$$
\begin{array}{cl}
\text { minimize } & V\left(d(t)+\mu(d(t)-d(t-1))^{2}\right) \\
+ & Q_{1}(t) m(t) q \\
\text { subject to } \quad & 0 \leq m(t) \leq A_{\max } .
\end{array}
$$

Since the distortion of the previous time slot, $d(t-1)$, is known, the objective function of problem (36) is solely dependent on $m(t)$. We can set the derivative of the objective function to 0 , i.e.,

$$
\begin{aligned}
& 2 \alpha \beta V(2 \mu \gamma+(1-2 \mu) d(t-1)) m^{\beta-1} \\
& +2 \alpha^{2} \beta V \mu m^{2 \beta-1}+Q_{1}(t) q=0,
\end{aligned}
$$

to obtain the optimal rate value. 
The second problem is for scheduling and power control.

(P7) minimize $\quad H=-\sum_{k=1}^{2} Q_{k}(t) b_{k}(t)+Z(t) y(t)$,

subject to $e_{k}(t)=\{0,1\}, e_{1}(t)+e_{2}(t) \leq 1$,

$$
0 \leq p(t) \leq p_{\max } \text {. }
$$

Rearranging the objective function and removing the fixed term $-Z(t) \bar{p}$, we have

$$
\begin{aligned}
H & =-\frac{\eta B}{F} \sum_{k=1}^{2} Q_{k}(t) e_{k}(t) \log \left(1+p(t) \frac{d^{-\alpha} G}{N_{0} B}|h(t)|^{2}\right) \\
& +\frac{1}{F} Z(t)\left(e_{1}(t)+e_{2}(t)\right) p(t) .
\end{aligned}
$$

To solve problem (38), we consider the three scheduling decisions separately, i.e., $e(t)=\{0,1\},\{1,0\}$ and $\{0,0\}$. When $e(t)=\{0,0\}, H=0$ and $p(t)=0$. For the other strategies, we have

$$
H=-\frac{\eta B}{F} Q_{k}(t) \log \left(1+p(t) \frac{d^{-\alpha} G}{N_{0} B}|h(t)|^{2}\right)+\frac{1}{F} Z(t) p(t) .
$$

We can set the gradient of (40) to zero to obtain the minimizer

$$
p(t)=\frac{\eta B Q_{k}(t)}{Z(t) \ln 2}-\frac{d^{\alpha} N_{0} B}{G|h(t)|^{2}}
$$

then we can choose the scheduling strategy and $p(t)$ which minimize $H$. The algorithm for each time slot is summarized as follows.

Step 1:Solve equation (37) to obtain $m(t)$. If $m(t)$ is out of the range of $\left[0, A_{\max }\right]$, set $m(t)$ to the nearest boundary.

Step 2:For all 3 possible scheduling strategies, solve (41) to obtain $p(t)$. If $p(t)$ is out of the range of $\left[0, p_{\max }\right]$, set $p(t)$ to the nearest boundary. Then use $p(t)$ to calculate $H$ based on (40). Choose the scheduling strategy and $p(t)$ which minimize $H$.

For problem (P4), similarly, two virtual queues can be built to satisfy the distortion and distortion variation constraints. Specifically, we define the following two virtual queues

$$
\begin{aligned}
& Z_{1}(t+1)=\max \left[Z_{1}(t)+y_{1}(t), 0\right], \\
& Z_{2}(t+1)=\max \left[Z_{2}(t)+y_{2}(t), 0\right],
\end{aligned}
$$

where $y_{1}(t)=d(t)-\bar{d}$ and $y_{2}(t)=(d(t)-d(t-1))^{2}-$ $\bar{d}_{v}$. Similar to problem (P3), we minimize the right-hand-side bound of (31), then decompose the problem into power control and rate control problem and solve them separately. As the approach is similar to that for problem (P3), due to the space limitation, we do not include the full details in this paper.

\section{Evaluation}

In this section, we evaluate the performance of the proposed rate-distortion model and the joint rate control, scheduling and power control algorithm. We adopted compressive sensing components similar as [18]. The structurally random matrix [19] is chosen as the measurement matrix, Undecimated
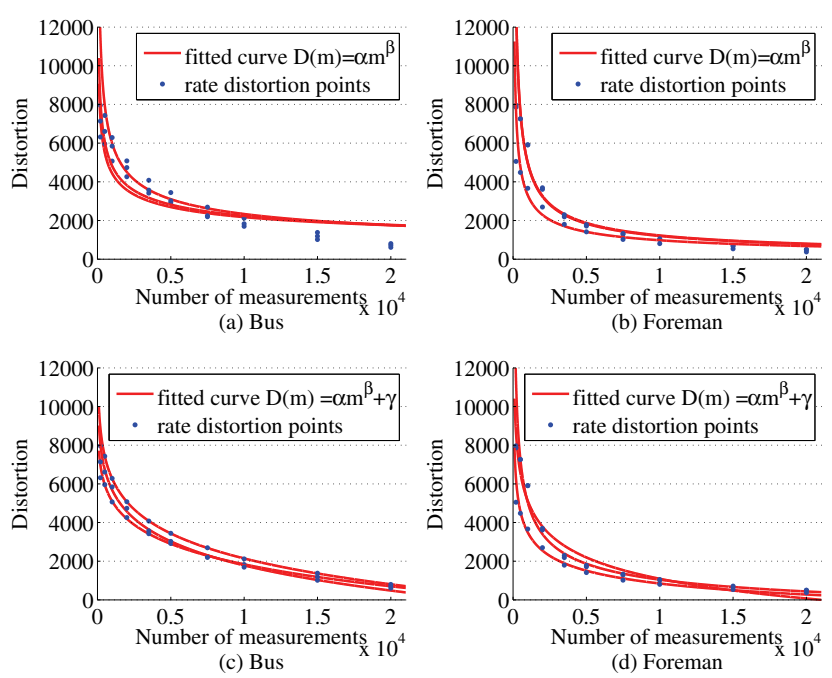

Fig. 2. Model comparison.

wavelet transform (UWT) is chosen as the basis, and analysisbased $\ell_{1}$ minimization is used to recover the signal from the measurements.

\section{A. Rate-Distortion Model}

Two video sequences "Bus" and "Foreman" are used to test how well the model capture the rate-distortion characteristic. The resolution of the two videos are $176 \times 144$ and the frame rate is 15 frames per second. For each video sequence, we randomly selected 3 frames and obtain the rate-distortion curve for each frame using model (7) and (8), respectively. Specifically, we generate 10 sets of different number of measurements for each frame: 200, 500,1000, 2000,3500, $5000,7500,10000,15000$, and 20000 . We set the number of bits for each measurement to 6 . We recover the images from these measurements and calculate the distortion, so we can obtain 10 rate-distortion points for each frame. We then use these rate-distortion points to fit the models. Figs. 2 (a) and (b) show the rate-distortion relationship captured by (7) for the two video sequences; (c) and (d) show the results using model (8) for the two video sequences. We can see that model (8) can capture the rate-distortion characteristic, because the extra parameter $\gamma$ brings one more degree of freedom. Other videos are tested and also show that model (8) is better than model (7). In the following, we adopt model (8) for the ratedistortion estimation. The proposed model can capture the rate-distortion relationship of all the frames of various types of videos. However, the parameters of the model may vary from frame to frame depending on the characteristic of the video. We can obtain the model parameter for each frame, but it suffers from high computational cost. Therefore, to reduce the computational cost in obtaining the parameters of the model, we used the same set of parameters for $F=15$ consecutive frames to explore the temporal redundancy of these frames. The value of $F$ can be larger for video surveillance applications where the videos are less dynamic. The distortion is calculated using $\|x-\hat{x}\|_{\ell_{2}}$, where $x$ is the original image signal and $\hat{x}$ is the reconstructed image signal. It is converted to PSNR as PSNR $=10 \times \log _{10}\left(\frac{255 \times 255 \times H \times W}{\|x-\hat{x}\| \ell_{2}}\right)$, where $H$ and $W$ are the height and width of the image, respectively. 
TABLE I

PROBLEM P3, POWER CONSTRAINT, $\bar{p}=0.8$ WATT

\begin{tabular}{|c|c|c|c|c|c|c|}
\hline V & $\begin{array}{c}\text { Avg. } \\
\text { distortion } \\
(\text { PSNR })\end{array}$ & $\begin{array}{c}\text { Avg. } \\
\text { distortion } \\
\text { variation } \\
(\mathrm{dB})\end{array}$ & $\begin{array}{c}\text { Avg. Q1 } \\
\text { backlog } \\
\text { (packets) }\end{array}$ & $\begin{array}{c}\text { Avg. Q2 } \\
\text { backlog } \\
\text { packets) }\end{array}$ & $\begin{array}{c}\text { Avg. Q1 } \\
\text { arrival rate } \\
\text { (kbits) }\end{array}$ & $\begin{array}{c}\text { Avg. } \\
\text { power }\end{array}$ \\
\hline 1 & $\begin{array}{c}7209.67 \\
(15.01)\end{array}$ & $\begin{array}{c}308.32 \\
(0.42)\end{array}$ & 0.58 & 0.31 & 2.64 & 0.65 \\
\hline 10 & $\begin{array}{c}5486.04 \\
(17.38)\end{array}$ & $\begin{array}{c}167.60 \\
(0.29)\end{array}$ & 1.64 & 0.75 & 8.25 & 0.74 \\
\hline $10^{2}$ & $\begin{array}{c}3767.88 \\
(20.65)\end{array}$ & $\begin{array}{c}98.99 \\
(0.23)\end{array}$ & 4.55 & 2.01 & 23.26 & 0.79 \\
\hline $10^{3}$ & $\begin{array}{c}2209.03 \\
(25.29)\end{array}$ & $\begin{array}{c}57.97 \\
(0.23)\end{array}$ & 12.44 & 4.57 & 55.09 & 0.80 \\
\hline $10^{4}$ & $\begin{array}{c}1529.25 \\
(28.48)\end{array}$ & $\begin{array}{c}42.02 \\
(0.23)\end{array}$ & 63.04 & 17.92 & 78.75 & 0.80 \\
\hline
\end{tabular}

\section{B. Algorithm Evaluation}

In this section, we evaluate the performance of the joint rate control, scheduling and power control algorithm. We consider a wireless transmission system with $B=600 \mathrm{KHz}$ and transmission power $0 \leq p \leq 1$ Watt. We set the reference SNR to $4.75 \mathrm{~dB}$ when the transmission power is 0.5 Watt, and $\gamma_{0}=\frac{d^{-\alpha} G}{N_{0} B}=5.97$. The transceiver efficiency $\eta$ is set to 0.9 . We assume that the arrival rate of the aggregated traffic from the non-video sensing data $a_{2}(t)$ confirms to the Poisson distribution with the arrival rate of 0.1 packet per time slot and the packet size is 6 kbits.

For the deterministic control algorithm, the average power consumption is set to 0.8 Watt. The total number of time slots is 5000. In order to reduce the complexity of the rate control algorithm, we perform rate allocation for every group of 50 slots and the parameter $M_{b}$ is the summation of the transmission rate of the corresponding slots. Meanwhile, the number of all possible measurements per frame is reduced from 20000 to 40 with an interval of 500 measurements. The results of the algorithm are as follows. The average distortion is $1502.86(28.63 \mathrm{~dB})$, the average distortion variation is 30.84 $(0.18 \mathrm{~dB})$, the average power consumption is 0.8 Watt.

For problem (P3), we set the time average power $\bar{p}$ to 0.8 Watt. On each time slot, we perform the control decisions obtained using Lyapounov optimization.

Table I shows the simulation results. When $V$ is larger, the flow control algorithm will admit more measurements into $Q_{1}$, as the average number of bits increases from 2.64 to 78.75 kbits. When $V$ is sufficiently large, the algorithm fully utilize the channel transmission rate and achieve minimum distortion. Since the number of measurements per frame is increased, the average distortion is reduced. Because the tradeoff between queueing delay and performance of min drift-pluspenalty algorithm, the average queue backlog of $Q_{1}$ and $Q_{2}$ increased from 0.58 to 63.04 and from 0.31 to 17.92 packets, respectively. The virtual queue $Z(t)$ is stable, so the average power consumption is less than or equal to the desired value $0.8 \mathrm{~W}$. Compared with the results of the upper bound (with the deterministic control algorithm), as $V$ is increased, the distortion and distortion variation are approaching to the optimal value of the deterministic algorithm, which demonstrate the
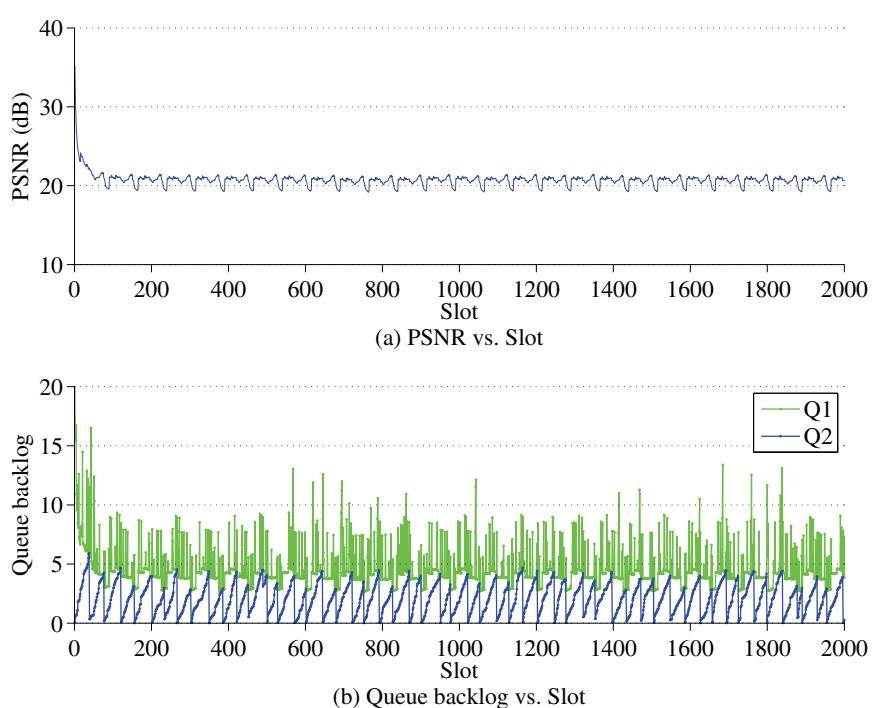

Fig. 3. $P 3$ trace $V=100$.

TABLE II

PROBLEM P4, DISTORTION CONSTRAINT, $\bar{d}=2000, \overline{d_{v}}=38.72$

\begin{tabular}{|c|c|c|c|c|c|c|}
\hline $\mathrm{V}$ & $\begin{array}{c}\text { Avg. } \\
\text { distortion } \\
(\text { PSNR) }\end{array}$ & $\begin{array}{c}\text { Avg. } \\
\text { distortion } \\
\text { variation }\end{array}$ & $\begin{array}{c}\text { Avg. Q1 } \\
\text { backlog } \\
\text { (packets) }\end{array}$ & $\begin{array}{c}\text { Avg. Q2 } \\
\text { backlog } \\
\text { (packets) }\end{array}$ & $\begin{array}{c}\text { Avg. Q1 } \\
\text { arrival rate } \\
\text { (kbits) }\end{array}$ & $\begin{array}{l}\text { Avg. } \\
\text { power }\end{array}$ \\
\hline $10^{4}$ & $\begin{array}{c}1998.43 \\
(26.16)\end{array}$ & $\begin{array}{c}65.95 \\
(0.3)\end{array}$ & 14.30 & 5.61 & 61.98 & 1.00 \\
\hline $5 \times 10^{6}$ & $\begin{array}{c}1998.87 \\
(26.15)\end{array}$ & $\begin{array}{c}65.80 \\
(0.29)\end{array}$ & 15.39 & 5.60 & 62.02 & 0.88 \\
\hline $10^{8}$ & $\begin{array}{c}1999.26 \\
(26.15)\end{array}$ & $\begin{array}{c}65.28 \\
(0.29)\end{array}$ & 17.70 & 5.82 & 61.74 & 0.69 \\
\hline
\end{tabular}

effectiveness of the stochastic algorithm.

Fig. 3 shows the simulation trace of the power constraint problem (P3) for $V=100$. Figs. 3 (a) and (b) show the PSNR vs. slot and queue backlog vs. slot, respectively. The total number of slots of the simulation is 5000 , and the figures show the first 2000 slots as the remaining slots have the similar pattern. The average number of measurements is 3876.7 and the average PSNR is $20.65 \mathrm{~dB}$. From the figure, the actual queues are stable and their average queue backlog are listed in Table I. At the beginning of the simulation, backlog of $Q_{2}$ is increasing, because the scheduling algorithm always choose $Q_{1}$ to transmit or transmit nothing until the queue backlog of $Q_{1}$ and $Q_{2}$ are similar. During this time interval, the backlog of $Q_{1}$ is much larger than that of $Q_{2}$, so it is more urgent to reduce the queue backlog of $Q_{1}$. Then this pattern continues. The trace figures for other values of $V$ are similar.

For problem $(\mathrm{P} 4)$, the maximum time average expected distortion $\bar{d}$ is set to $2000(26.15 \mathrm{~dB})$, and $\bar{d}_{v}$ is set to 1500 . The objective is to minimize power consumption.

The simulation results are shown in Table V-B. When $V$ is increased from $10^{4}$ to $10^{8}$, the average distortion is approaching the desired average distortion and the average power is reduced from 1 to 0.69 Watt. Due to the trade- 

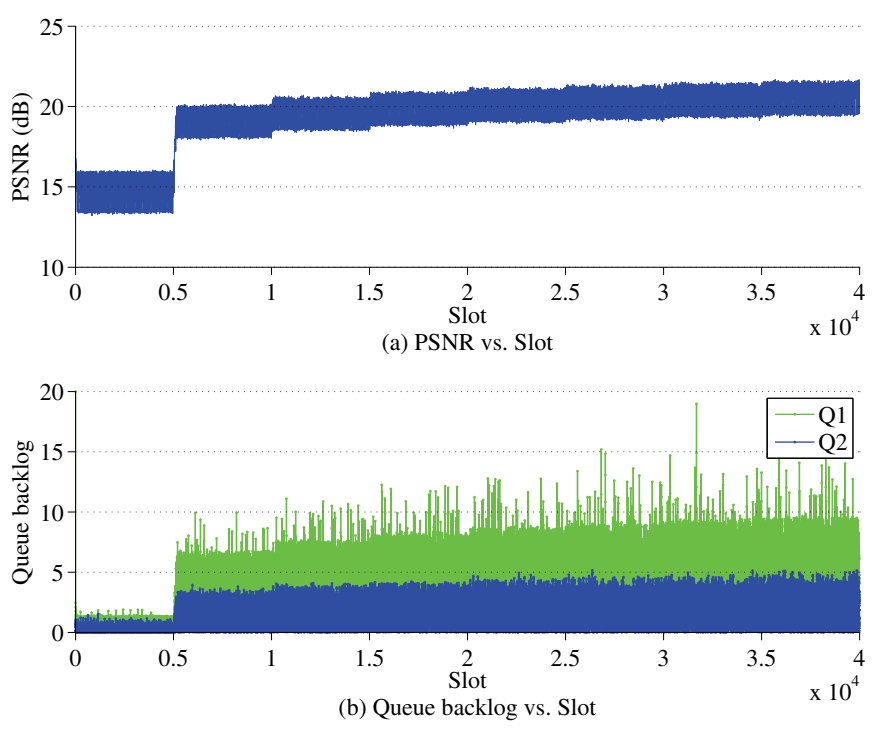

Fig. 4. Incremental-V algorithm trace, $V=135.89$.

off between the queueing delay and the performance of min drift-plus-penalty algorithm, the average queue backlog of $Q_{1}$ and $Q_{2}$ increased from 14.30 to 17.7 and from 5.61 to 5.82 packets, respectively. The virtual queues are stable, so the time average expected distortion $\bar{d}$ is smaller than 2000. The distortion variation is larger than the target 38.72 . It is because we update the rate-distortion model every 15 frames, which leads to the inaccuracy. However, the distortion variation is still acceptable.

\section{Incremental-V algorithm}

From the previous section, we can see that when increasing $\mathrm{V}$, the objective function is pushed to the optimal value but the average queue length and average queueing delay is increased as well. For video transmission applications, we need to control the queueing delay. In this section, we propose a variant algorithm to achieve the above goal. We increase the value of $V$ every $T$ time slots according to

$$
V(k)=V_{0}(1+k T)^{\sigma},
$$

where $V_{0}$ is the initial value of $V$ and $\sigma$ controls the growing speed of $V$. [17] has shown that the Lyapunov optimization algorithm can achieve optimality when increasing $V$ according to the above equation every time slot. However, for the purpose of controlling the average delay, we increase $V$ until the average transmission delay during the last $T$ time slots reaches the target delay $t_{d}$.

To evaluate the algorithm, we set $T$ to 5000 time slots, $\sigma$ to 0.45 , and $t_{d}$ to $400 \mathrm{~ms}$. Fig. 4 shows the trace of the incremental-V algorithm, where (a) and (b) show the PSNR vs. slot and queue backlog vs. slot, respectively. From the figure, the PSNR and queue length increase in a staircase manner until the average transmission delay reaches $t_{d} . V$ is increased and stopped at 135.9 , the average video distortion is 3728.08 (20.74 dB); average queue length of $Q_{1}$ and $Q_{2}$ are 4.86 and 2.1 kbits, respectively; and the average power consumption is $0.79 \mathrm{~W}$. In this way, we can push the objective function to the optimal value while maintaining the average transmission delay within an acceptable range.

The incremental- $\mathrm{V}$ algorithm shows the same convergence property and performance bound in each time period $T$ as the previous algorithms, since the value of $V$ is fixed for each time period. The number of time periods needed before the convergence of the algorithm to the target transmission delay depends on parameter $V_{0}$ and $\sigma$ in (44). If we choose a larger value of $V$ and $\sigma$, the step size of $V$ is larger and the value of $V$ is increased quickly, and thus it may increase the convergence speed. However, it may also lead to a longer transmission delay than expected. How to choose $V_{0}$ and $\sigma$ to reduce convergence time is left for future exploration.

\section{CONCLUSION}

In this paper, we have proposed a simple and effective rate-distortion model for compressive sensed video. Using this model, we can accurately estimate the video distortion given the number of measurements used in signal recovery. We have formulated a deterministic optimization problem as a bench mark, and a more practical stochastic optimization problem for transmitting compressive sensing video over wireless channels. A supplementary stochastic optimization problem has been formulated to consider distortion and power consumption with different priority. We have further proposed an incremental- $V$ algorithm, which helps to choose the value of $V$ while keeping the average transmission delay in a reasonable range. The simulation results have shown the effectiveness of the proposed transmission control algorithms. When solving the stochastic optimization problems, we assume that the channel fading coefficient is known for the current slot. In practice, channel condition can be estimated through feedback from the receiver. The impact of the delayed and inaccurate channel estimation is left for future exploration. For simplicity, we used channel capacity to calculate the transmission rate based on channel condition and power level. In reality, the transmission rate also depends on the modulation and coding schemes adopted in the system. How to extend this work to consider discrete link rates requires further investigation.

\section{REFERENCES}

[1] M. F. Duarte, M. A. Davenport, D. Takhar, J. N. Laska, T. Sun, K. F. Kelly, and R. G. Baraniuk, "Single-pixel imaging via compressive sampling," IEEE Signal Process. Mag., vol. 25, no. 2, pp. 83-91, Mar. 2008.

[2] D. L. Donoho, "Compressed sensing," IEEE Trans. Inf. Theory, vol. 52, no. 4, pp. 1289-1306, Apr. 2006.

[3] E. J. Candes and T. Tao, "Near-optimal signal recovery from random projections: universal encoding strategies?" IEEE Trans. Inf. Theory, vol. 52, no. 12, pp. 5406-5425, Dec. 2006.

[4] J. Xu, X. Shen, J. W. Mark, and J. Cai, "Adaptive transmission of multilayered video over wireless fading channels," IEEE Trans. Wireless Commun., vol. 6, no. 6, pp. 2305-2314, June 2007.

[5] J. Zhang, J. Chen, and Y. Sun, "Transmission power adjustment of wireless sensor networks using fuzzy control algorithm," Wireless Commun. and Mobile Comput., vol. 9, no. 6, pp. 805-818, 2009.

[6] R. Zhang, R. Ruby, J. Pan, L. Cai, and X. Shen, "A hybrid reservation/contention-based MAC for video streaming over wireless networks," IEEE J. Sel. Areas Commun., vol. 28, no. 3, pp. 389-398, Apr. 2010.

[7] L. Cai, S. Xiang, Y. Luo, and J. Pan, "Scalable modulation for video transmission in wireless networks," IEEE Trans. Veh. Technol., vol. 60, no. 9, pp. 4314-4323, Nov. 2011. 
[8] S. Xiang, L. Cai, and J. Pan, "Adaptive scalable video streaming in wireless networks," in Proc. 2012 ACM MMSys, pp. 167-172.

[9] A. Abdulla, H. Nishiyama, J. Yang, N. Ansari, and N. Kato, "Hymn: a novel hybrid multi-hop routing algorithm to improve the longevity of WSNs," IEEE Trans. Wireless Commun., vol. 11, no. 7, pp. 2531-2541, Jul. 2012.

[10] Z. Charbiwala, S. Chakraborty, S. Zahedi, Y. Kim, M. B. Srivastava, T. He, and C. Bisdikian, "Compressive oversampling for robust data transmission in sensor networks," in 2010 IEEE INFOCOM.

[11] J. N. Laska, P. Boufounos, M. A. Davenport, and R. G. Baraniuk, "Democracy in action: quantization, saturation, and compressive sensing," preprint, 2009.

[12] S. Pudlewski, A. Prasanna, and T. Melodia, "Compressed-sensingenabled video streaming for wireless multimedia sensor networks," IEEE Trans. Mobile Comput., vol. 11, no. 6, pp. 1060-1072, June 2012.

[13] K. Stuhlmuller, N. Farber, M. Link, and B. Girod, "Analysis of video transmission over lossy channels," IEEE J. Sel. Areas Commun., vol. 18 , no. 6, pp. 1012-1032, 2000.

[14] M. J. Neely and A. Sharma, "Dynamic data compression with distortion constraints for wireless transmission over a fading channel," Arxiv preprint arXiv:0807.3768, 2008.

[15] H. Everett III, "Generalized Lagrange multiplier method for solving problems of optimum allocation of resources," Operations Research, pp. 399-417, 1963.

[16] G. J. Sullivan and T. Wiegand, "Rate-distortion optimization for video compression," IEEE Signal Process. Mag., vol. 15, no. 6, pp. 74-90, Nov. 1998.

[17] M. J. Neely, "Stochastic network optimization with application to communication and queueing systems," Synthesis Lectures on Commun. Networks, vol. 3, no. 1, pp. 1-211, 2010.

[18] S. Xiang and L. Cai, "Scalable video coding with compressive sensing for wireless videocast," in 2011 IEEE ICC.

[19] T. Do, T. Tran, and L. Gan, "Fast compressive sampling with structurally random matrices," in Proc. 2008 IEEE ICASSP, pp. 3369-3372.

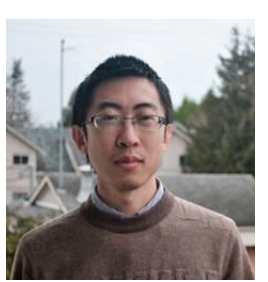

Siyuan Xiang (S'10) received the M.Eng. degree from Tongji University, Shanghai, China, in 2008. $\mathrm{He}$ is currently working toward the Ph.D. degree with the Department of Electrical and Computer Engineering, University of Victoria, Victoria, BC, Canada. His research interest is multimedia communications.

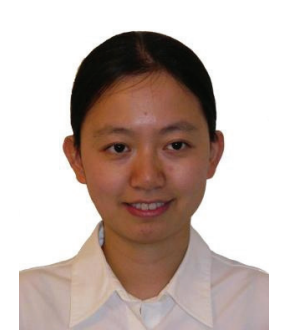

Lin Cai Lin Cai (S'00-M'06-SM'10) received her M.A.Sc. and $\mathrm{PhD}$ degrees (awarded Outstanding Achievement in Graduate Studies) in electrical and computer engineering from the University of Waterloo, Waterloo, Canada, in 2002 and 2005, respectively. Since 2005, she has been an Assistant Professor and then an Associate Professor with the Department of Electrical \& Computer Engineering at the University of Victoria. Her research interests span several areas in wireless communications and networking, with a focus on network protocol and architecture design supporting emerging multimedia traffic over wireless, mobile, ad hoc, and sensor networks.

She has been a recipient of the NSERC Discovery Accelerator Supplement Grant in 2010, the best paper award of IEEE ICC 2008, and the best academic paper award of IEEE WCNC 2011. She has served as a TPC symposium co-chair for IEEE Globecom'10 and IEEE Globecom'13, and the Associate Editor for IEEE TRANSACTIONS ON WIRELESS COMMUNICATIONS, IEEE TRANSACTIONS ON VeHICUlaR TECHNOLOGY, EURASIP Journal on Wireless Communications and Networking, International Journal of Sensor Networks, and Journal of Communications and Networks (JCN). 